\title{
SYSTEMS OF QUADRATICALLY COUPLED DIFFERENTIAL EQUATIONS WHICH CAN BE REDUCED TO LINEAR SYSTEMS
}

\author{
BY IRVIN KAY \\ Communicated by Fred Brauer, September 21, 1972
}

1. Introduction. Systems of ordinary differential equations with quadratic coupling have been used to model growth processes which occur in a number of otherwise unrelated physical applications (cf. [1], [2], [3]). Explicit solutions for the initial value problem have been obtained in certain cases when the coupling coefficients have been appropriately specialized (cf. [2], [3], [4]). This paper will consider a somewhat more general class of quadratically coupled systems for which the initial value problem can be reduced to that of a linear system.

2. Conditions for the reduction. The most general system of quadratically coupled differential equations over the complex field can be expressed in the form

$$
\dot{x}^{i}+\sum_{j, k=1}^{n} \Gamma_{j k}^{i} x^{j} x^{k}+\sum_{j=1}^{n} A_{j}^{i} x^{j}=b^{i}, \quad i=1, \ldots, n .
$$

If the coefficients in (1) are constant and satisfy the relations

$$
\sum_{j=1}^{n} \Gamma_{j k}^{i} \Gamma_{l m}^{j}=\sum_{j=1}^{n} \Gamma_{l j}^{i} \Gamma_{m k}^{j},
$$

and either

$$
A_{j}^{i}=\sum_{k=1}^{n} \Gamma_{j k}^{i} a^{k}
$$

or

$$
A_{j}^{i}=\sum_{k=1}^{n} \Gamma_{k j}^{i} a^{k},
$$

where the $a^{k}$ are the components of some constant vector $a$, then the solution to the initial value problem for (1) can be reduced to that for a linear system with constant coefficients.

The requirement $(2 a)$ is the necessary and sufficient condition that the $\Gamma_{j k}^{i}$ be the structure constants for an $n$ dimensional algebra (cf. [5]). In particular, the $n$ matrices $\Gamma_{k}, k=1, \ldots, n$, whose elements are $\Gamma_{j k}^{i}$, them-

AMS(MOS) subject classifications (1970). Primary 34A05; Secondary 34A30, 15A24, $15 \mathrm{~A} 30$. 
selves, form a basis for such an algebra. The reduction of (1) to a linear system can be generalized to the case of nonconstant coefficients if, in addition to the condition (2), the condition that the $\Gamma_{k}$ satisfy the differential equations

$$
\dot{\Gamma}_{k}+G \Gamma_{k}=0, \quad k=1, \ldots, n,
$$

for some matrix $G$, is also required.

THEOREM 1. If a multiplication law

$$
\{z=x y\} \stackrel{\text { def }}{\equiv}\left\{z^{i}=\sum_{j, k=1}^{n} \Gamma_{j k}^{i} x^{j} y^{k}, i=1, \ldots, n\right\}
$$

is defined for vectors $x$ with elements $x^{i}$ over the complex field, the resulting algebra of vectors $x$ will be associative iff (2a) is satisfied.

COROLlaRY. The algebra defined by (4) will be commutative iff $\Gamma_{j k}^{i}=$ $\Gamma_{k j}^{i}, i, j, k=1, \ldots, n$.

3. Reduction to a linear system. If (1) is multiplied through by $\Gamma_{m i}^{l}$ and summed over $i$, and (2) is taken into account, the result will have the form

$$
\dot{X}+X^{2}+A X=B
$$

or

$$
\dot{X}+X^{2}+X A=B
$$

where $X$ is the matrix whose elements are

$$
X_{j}^{i}=\sum_{k=1}^{n} \Gamma_{j k}^{i} x^{k}
$$

$A$ is the matrix whose elements are $A_{j}^{i}$, and $B$ is the matrix whose elements are

$$
B_{j}^{i}=\sum_{k=1}^{n} \Gamma_{j k}^{i} b^{k}
$$

The matrix differential equation (5) has the form of a Riccati equation and therefore can be reduced to linear form by means of the substitution (cf. $[6])$ :

$$
X=\dot{W} W^{-1} \quad \text { or } \quad X=W^{-1} \dot{W} .
$$

The initial value problem for equation (5) is then reduced to that for a linear second order differential equation for the nonsingular matrix $W$ :

$$
\ddot{W}+A \dot{W}=B W \text { or } \ddot{W}+\dot{W} A=W B \text {. }
$$


If initial values for (5) are chosen consistent with conditions (2), it can be shown with the aid of standard existence theorems that the solution of the initial value problem for (5) will necessarily have the form (6). In general, the solution of (6) can be expressed in the form

$$
x^{i}=y^{i}+a^{i}, \quad i=1, \ldots, n,
$$

where $y^{i}$ is any particular solution of (6) and $a^{i}$ satisfies

$$
\sum_{k=1}^{n} \Gamma_{j k}^{i} a^{k}=0, \quad i, j=1, \ldots, n
$$

Then, if the expression for $x^{i}$ given by (7) is substituted into (1), with the aid of (8) a linear differential equation for $a^{i}$ is obtained. The appropriate initial condition for this equation depends on the choice of $y^{i}$ initially. The differential equation for $a^{i}$ in general will not have constant coefficients unless the matrices $\Gamma_{k}$ whose elements are $\Gamma_{j k}^{i}$ form a linearly independent set, in which case the $a^{i}$ are identically zero. Thus, by analogy with linear systems, it may be concluded that a kind of resonance phenomenon occurs when the $\Gamma_{k}$ are linearly dependent. ${ }^{1}$

4. Some useful relations. The following (Theorem 2 ) may help to simplify the task of determining whether the condition (2a) holds in a given application. Theorem 3 demonstrates that the linearization procedure leads to a system which is completely uncoupled in certain cases.

Since $\Gamma_{j k}^{i}$ in (1) is determined only up to a three index symbol $S_{j k}^{i}$ which is symmetric in its lower indices, the possibility exists that some antisymmetric symbol $A_{j k}^{i}$ can be added to satisfy the condition (2a). It is, therefore, useful to obtain a simple restriction on the antisymmetric part of $\Gamma_{j k}^{i}$, which, according to the Corollary to Theorem 1, cannot be symmetric in its lower indices unless the algebra defined by the multiplication law (4) is commutative.

THEOREM 2. A necessary condition for $\Gamma_{j k}^{i}$ to satisfy (2a) is that

$$
\sum_{j=1}^{n} A_{j k}^{i} S_{k k}^{j}=0, \quad i, k=1, \ldots, n,
$$

where

$$
S_{j k}^{i}=\frac{1}{2}\left(\Gamma_{j k}^{i}+\Gamma_{k j}^{i}\right) \quad \text { and } \quad A_{j k}^{i}=\frac{1}{2}\left(\Gamma_{j k}^{i}-\Gamma_{k j}^{i}\right) .
$$

THEOREM 3. If (2a) is satisfied, the $\Gamma_{j k}^{i}$ are symmetric in their lower indices, and the matrices $\Gamma_{k}$, whose elements are $\Gamma_{j k}^{i}$, are all diagonalizable, then the $\Gamma_{j k}^{i}$ will have the canonical form

\footnotetext{
${ }^{1}$ This paragraph resulted directly from a question posed by the referee.
} 


$$
\Gamma_{j k}^{i}=\sum_{l=1}^{n} H_{l}^{i} G_{j}^{l} G_{k}^{l}, \quad i, j, k=1, \ldots, n .
$$

The $G_{j}^{i}$ are elements of some matrix $G$ and the $H_{j}^{i}$ are elements of a matrix $H$ such that the product $G H$ satisfies the relation $G H G_{k}=G_{k}, k=1, \ldots, n$, where the $G_{k}$ are matrices whose elements $G_{j k}^{i}$ are given by $G_{j k}^{i}=G_{j}^{i} G_{k}^{i}$. In particular, if $G$ is nonsingular $H=G^{-1}$.

When Theorem 3 holds along with the remaining condition (2b) or (2c) the linearization procedure leads to an uncoupled system of $n$ independent scalar Riccati equations and, therefore, to $n$ independent, linear scalar, ordinary differential equations.

The author wishes to express his gratitude to the referee for his careful reading of this paper, for his interesting comments, one of which led to a significant improvement in the results, and for supplying references [7] and [8]. The referee made the observation that L. Marcus in [7] associated a generally nonassociative algebra with a quadratically coupled system of differential equations that is completely equivalent to the algebra defined in this paper through the multiplication law (4). J. J. Levin and S. S. Shatz, with the aid of results given by Marcus, considered a somewhat different relationship between matrix Riccati equations and quadratically coupled equations than is done in this paper.

The referee also observed the following apparent paradox: If $\Gamma$ is a constant $m \times n$ matrix, then the matrix Riccati equation

$$
d X / d t=X \Gamma X,
$$

where $X$ is $n \times m$, is a special case of

$$
\dot{x}^{i}=\sum_{j, k} a_{j, k}^{i} x^{j} x^{k} .
$$

Levin and Shatz show that the Markus algebra associated with (9) is isomorphic to the algebra of all $n \times m$ matrices with multiplication defined by

$$
A \cdot B=\frac{1}{2}(A \Gamma B+B \Gamma A) .
$$

It is important to note that (11), even with $n=m$, is in general not associative. Since (2a) (see Theorem 1) is satisfied if and only if the Markus algebra for (11) is associative, it follows that the procedure given in this paper transforming certain equations of type (1) into matrix Riccati equations fails in general for equations of type (9). This is a curious and interesting fact.

\section{REFERENCES}

1. E. R. Fisher and R. H. Kummler, Relaxation by vibration-vibration exchange processes, J. Chem. Phys. 49 (1968), 1075-1084. 
2. N. S. Goel, S. C. Maitra and E. W. Montroll, On the Volterra and other nonlinear models of interacting populations, Rev. Mod. Phys. 43 (1971), 231-276.

3. E. W. Montroll, "Stochastic Processes and Chemical Kinetics," in Energetics in metallurgical phenomena. Vol. 3, Gordon and Breach, New York, 1967, pp. 125-187.

4. K. E. Shuler, Exactly solvable nonlinear relaxation processes. Systems of coupled harmonic oscillators, J. Chem. Phys. 45 (1966), 1105-1110.

5. L. Rédei, Algebra. Vol. I, Akad. Kiadó, Budapest, 1954; English transl., Pergamon Press, New York, 1967, p. 243. MR 16, 559; MR 35 \# 2697.

6. J. J. Levin, On the matrix Riccati equation, Proc. Amer. Math. Soc. 10 (1959), 519-524. MR 21 \# 7344.

7. L. Markus, Quadratic differential equations and nonassociative algebras. Contributions to the theory of nonlinear oscillations. Vol. 5, Princeton Univ. Press, Princeton, N.J., 1960, pp. 185-213. MR 24 \# A2580.

8. J. J. Levin and S. S. Shatz, Riccati algebras, Duke Math. J. 30 (1963), 579-594. MR 28 \#3069.

Department of Electrical Engineering, Wayne State University, Detroit, MichiGAN 48202 .

Current address: Institute for Defense Analysis, 400 Army Navy Drive, Arlington, Virginia 22202 